\title{
A Compact Multiband Metamaterial based Microstrip Patch Antenna for Wireless communication Applications
}

\author{
Nikhil Kulkarni*, G. B. Lohiya** \\ *(Department of Electronic and Telecommun ication, Mu mbai University, India \\ Email: kulnik27@yahoo.in) \\ ** (Department of Electronic and Teleco mmun ication, Mu mbai University, India \\ Email: share.lgin fo@g mail.com)
}

\begin{abstract}
In this paper, a metamaterial based compact multiband microstrip antenna is proposed which can give high gain and directivity. Metamaterials are periodic structures and have been intensively investigated due to the particular features such as ultra-refraction phenomenon and negative permittivity and/or permeability. A metamaterialbased microstrip patch antenna with enhanced characteristics and multi band operation will be investigated in this work. The multiple frequency operation will be achieved by varying the capacitance of the metamaterial structure with the help of metallic loadings placed in each metamaterial unit cells. The potential impacts will be miniaturization, reduced cost and reduced power consumption since multiple antennas operating at different frequencies are replaced by a single antenna which can operate at multiple frequencies. The proposed microstrip patch antenna will have its frequencies of operation in the $\mathrm{L}, \mathrm{S}$ and $\mathrm{C}$ bands. The proposed structure is simulated using Agilent Advanced Design System (ADS) 2011.05. It is then fabricated on the FR4 substrate and the performance of the fabricated antenna is measured using the Vector Network Analy zer (VNA).
\end{abstract}

Keywords - Metamaterial, Microstrip Antenna, Bandwidth, Tunability, Frequency Range, Gain

\section{Introduction}

In wireless applications different antennas are required for different applications [1]. This increases the size of the wireless communication. Also high gain and high bandwidth is needed in any communication systems. To achieve all these features simultaneously in a single device, the concept of metamaterial is used along with microstrip antenna (MSA) [2]-[5]. If the size of microstrip antenna is large, then it provides high gain and vice versa [2].

\section{B asic Microstrip Patch Antenna}

A microstrip patch antenna is one of the most commonly utilized printed antennas. It consists of a radiating patch on one side of a dielectric substrate and a ground plane on the other side. An MSA can be of any shape, but rectangular shape MSA is most commonly used. Microstrip patch antenna, in their most basic form, benefits from their low profile, low cost, simplicity, and omnidirectional radiation pattern. Narrow bandwidth is one of the main disadvantages of a microstrip patch antenna. The antenna has to operate over a wide bandwidth in case of multichannel applications. But at a given time it has to operate over only a small bandwidth to cover a single channel. Radiation from the MSA can occur from the fringing fields between the periphery of the patch and the ground plane. To enhance the fringing fields from the patch, which account for the radiation, the width $w$ of the patch is increased. The fringing fields are also enhanced by decreasing the $\varepsilon_{r}$ or by increasing the substrate thickness $h$ [1]. The basic structure of MSA is given in Fig. 1.

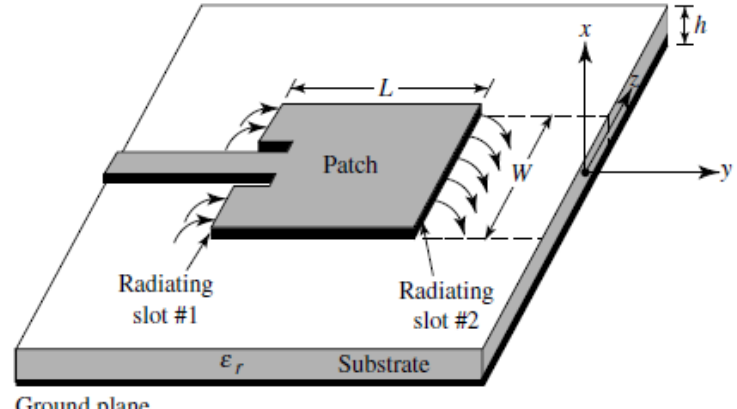

Figure 1 microstrip antenna

The width and length of MSA will be given by [6],

$w=\left[c / 2 f_{o}\left(\sqrt{ }\left(\varepsilon_{r}+1\right) / 2\right)\right]$

$\varepsilon_{e f f=}\left[\left(\varepsilon_{r}+1\right) / 2\right]+\left\{\left[\left(\varepsilon_{r}-1\right) / 2\right] *[1 / \sqrt{ } 1+\right.$

$12(h / w)]\}$

$l=\left(c / 2 f_{0} \sqrt{ } \varepsilon_{\text {eff }}\right)-0.824 \mathrm{~h}\left\{\left[\left(\varepsilon_{\text {eff }}+0.3\right) *((w / h)+\right.\right.$

$\left.0.264)] /\left[\left(\varepsilon_{\text {eff }}-0.258\right) *((w / h)+0.8)\right]\right\}$

... (3)

where,

- Velocity of light $(c)=3^{*} 10^{8} \mathrm{~m} / \mathrm{s}$,

- $f_{0}=$ Centre frequency $(\mathrm{GHz})$

- Dielectric constant $\left(\varepsilon_{\mathrm{r}}\right)=4.28$,

- $\varepsilon_{e f f}=$ Effective die lectric constant,

- $l=$ Length,

- $\quad w=$ Width and

- $\quad$ Height $(h)=1.57 \mathrm{~mm}$. 
Using equations (1), (2) and (3) the MSA design for center frequency $\left(f_{0}\right) 5 \mathrm{GH}$ for which

$l=13.76 \mathrm{~mm}$.

$\mathrm{w}=18.24 \mathrm{~mm}$.

and is shown in Fig. 2.

The simulation result of this microstrip patch antenna is given in Fig. 3.

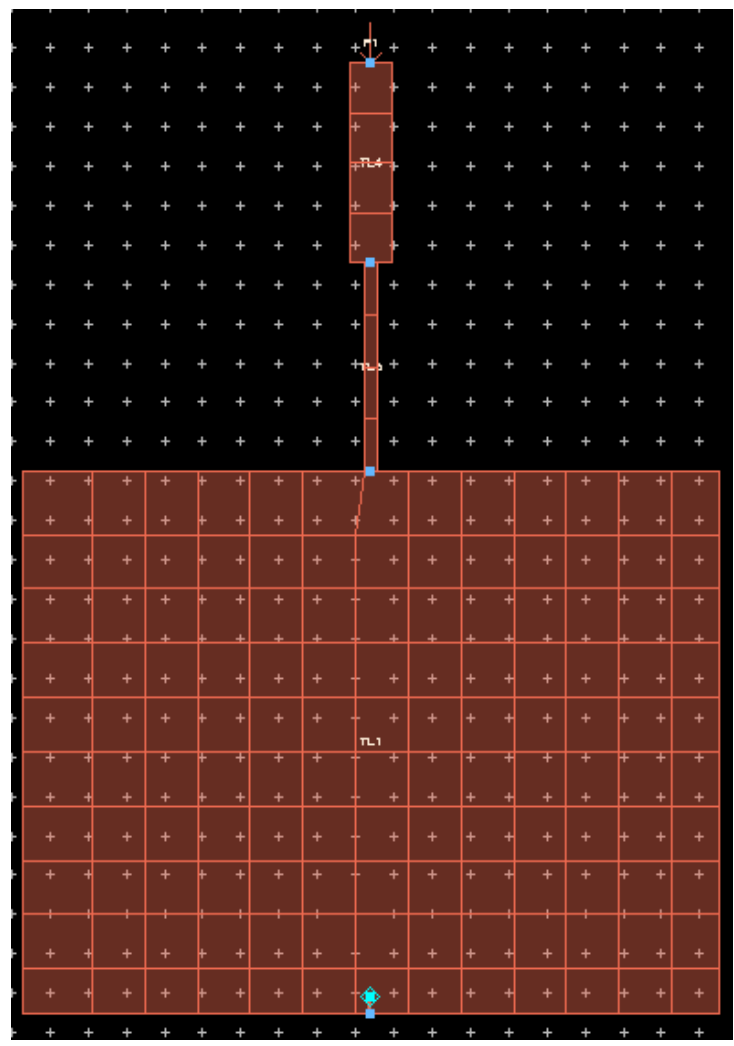

Figure 2 simu lation of mic rostrip antenna for $\mathrm{f}_{0}=5 \mathrm{GHz}$ in ADS
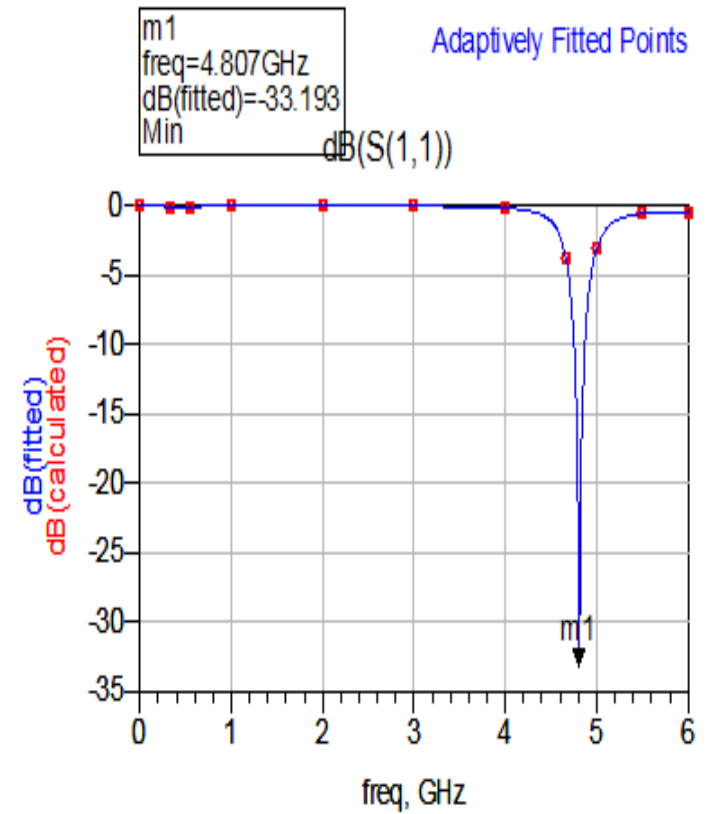

Figure 3 simu lation result output for $\mathrm{f}_{0}=5 \mathrm{GHz}$
The disadvantages of Microstrip Patch Antenna are:-

- Limited bandwidth (usually 1 to $5 \%$ ).

- Low gain.

- Low power handling.

To overcome this drawback metamaterial plays an important role [1]-[2].

\section{Me tamate rial}

Metamaterials are artificial materials synthesized by embedding specific inclusions in host media and they exhibit the properties of either negative permittivity or permeability. If both negative permittivity and negative permeability happen at the same time, then the composite exhibits an effective negative index of refraction and is referred to as left handed metamaterials (LHMs) [7]-[9]. 4 Permittivity, Permeability and Refractive Index graph for metamaterial is shown in Fig. 4

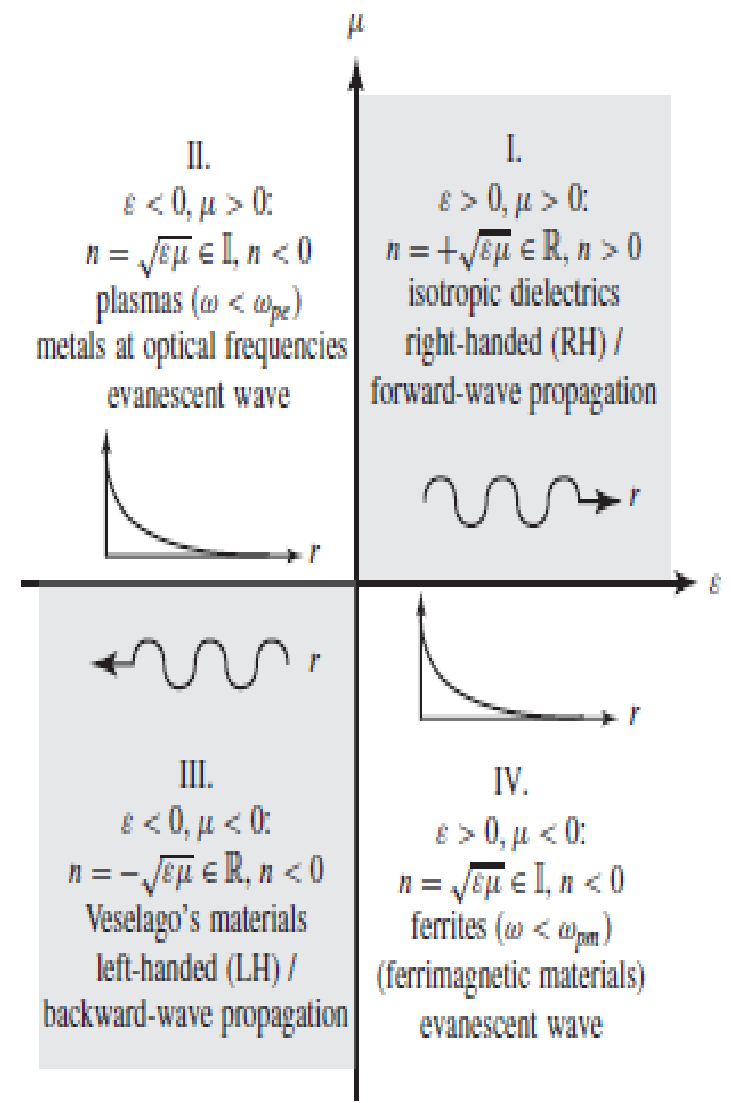

Figure 4 Permittivity, Permeability and Refractive Index graph

The idea of metamaterial was first proposed theoretically by Veselago in 1968 [7]. The negative permittivity was demonstrated and 
theorized with an array of metallic wires in 1996 by Pendry.

The structure had plasma frequency and thereby negative permittivity in the microwave regime. The structure of negative permeability was demonstrated and theorized in 1999 with split ring resonator (SRR) structure. The negative index of refraction existed in the region where both the real parts of the electric permittivity and magnetic permeability were simultaneously negative, typically in a structure composed of SRRs and metallic wires. Metamaterials exhibit exceptional properties not readily observed in nature. The inclusion of metamaterial in the design improves the characteristics of a microstrip patch antenna due to these exceptional properties. The first results of emission in metamaterial demonstrated, under proper conditions the energy radiated by a source embedded in a slab of metamaterial, concentrated in a narrow cone in the surrounding media. Metamaterials and its utilization for antenna's techniques were identified such as improvement of bandwidth, gain miniaturization, directivity and reduction of mutual coupling between elements in an antenna array. Enhanced bandwidth, high gain and high directivity were achieved using a metamaterial layer either as substrate or superstrate.

\subsection{Metamaterial SSR Structure}

The split ring resonator (SRR) structure as shown in Fig. 5 is the metamaterial substrate used as a superstrate on the microstrip patch for tuning. The outer ring of the structure measures $7 \mathrm{~mm}$ in length as well as the width. Similarly, the inner ring measures $5 \mathrm{~mm}$ in length and width. The opening measures $1 \mathrm{~mm}$ in width and $0.5 \mathrm{~mm}$ in length. Each microstrip patch has three SSR structure placed on it and then the antenna is simulated. The loadings placed in the SRR structure are small square measuring $0.5 \mathrm{~mm}$ each which helps in tuning [10].

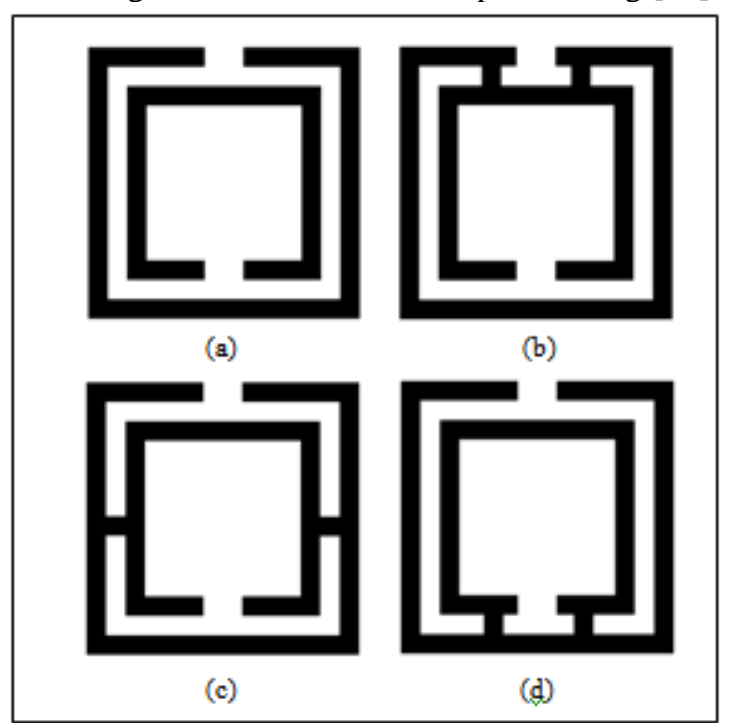

Figure 5 proposed metamaterial SSR structure a) metamaterial without load

b) metamaterial with loading 1

c) meta material with loading 2

d) metamaterial with loading 3

\subsection{Metamate rial-based Tunability}

Metamaterials find a lot of applications in devices, which are operating in mic rowave regime. To add flexibility and controllability to these devices, the researchers started working for tunable metamaterials [11]. The review is scrutinizing the methods, which can be integrated with a microstrip patch antennas. In the following way, the approaches to design tunable metamaterial is categorized, which can be incorporated in the design of a microstrip patch antennas:

- Tunability by incorporating varactor diode or PIN diodes in the metamaterial

- Tunability by varying the structure of SRR

\section{Metamate rial Based Multiband Microstrip Patch Antenna}

In conventional microstrip patch antenna, to achieve multiband operation different antennas are required [1]. In proposed metamaterial based multiband MSA, even a single patch out of the four patch in [12] can be used. The design of Metamaterial Based Microstrip Antennas is done in three parts: first the conventional MSA is simulated and the reconfigurability is tested, then the antenna is tested with metamaterial superstrate to have increase gain [3] and bandwidth [13]-[14] then the individual patches with metamaterial are simulated to test the multiband operation.

The proposed design of multiband microstrip patch antenna covers $\mathrm{L}, S$ and $C$ band in electromagnetic spectrum. To increase the gain and bandwidth, metamaterial is added to the antenna [5]. The base patch of microstrip antenna includes main radiator and four sub-patches placed on FR-4 substrate with relative permittivity $=4.4$ and height of a substrate $h=1.57 \mathrm{~mm}$. The metamaterial substrate is placed on top of this patch leaving an air gap of $1 \mathrm{~mm}$. The total volume of the microstrip antenna design is $50 \times 50 \times 1.57 \mathrm{~mm}^{3}$ [12]. Software used for simulation of antenna design is Agilent Advanced Design System (ADS) 2011.05. It supports Momentum as well as FEM mode of simulation.

As already stated, single slotted patch is sufficient enough for the multiband operation [12]. Further (using patch 2) simulation shown only with one MSA is shown in Fig. 6. The results of the simulation and measured results are shown in the Fig. 7 and 8 respectively. 


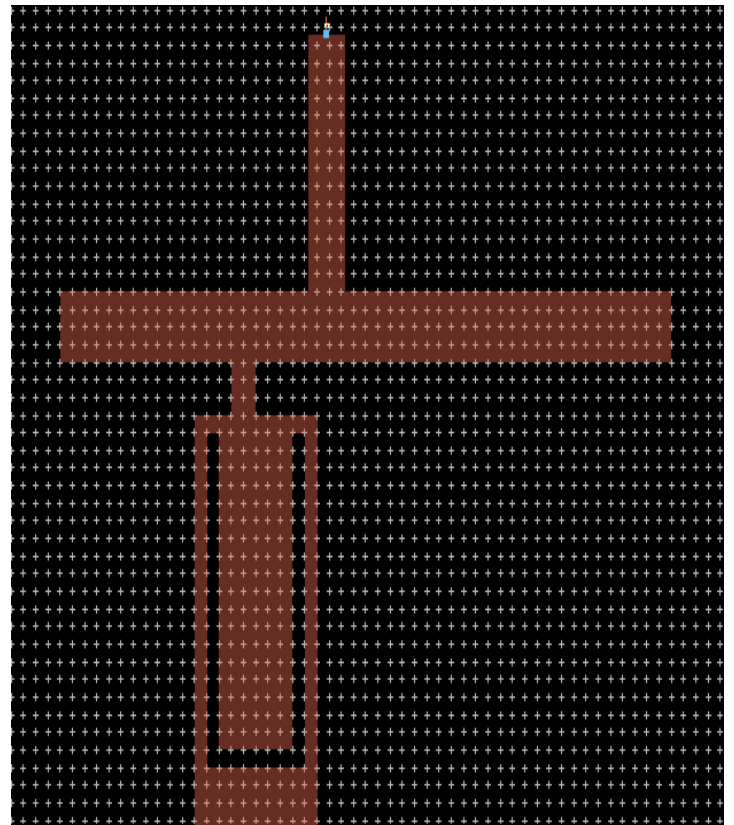

Figure 6 modified structure of sub-patch 2 in ADS software

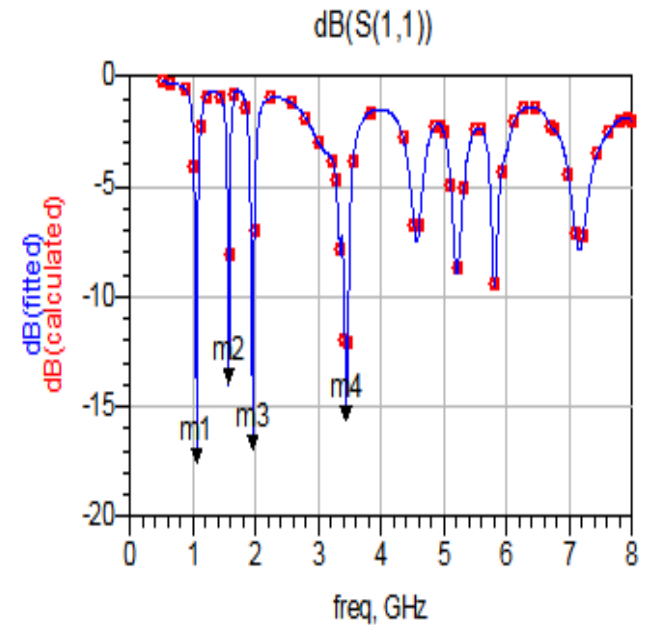

Figure 7 simu lation results of sub-patch 2

Table 1 Simu lated result for frequency and return loss of MSA sub patch 2

\begin{tabular}{|c|l|l|}
\hline & $\begin{array}{l}\text { Frequency } \\
(\mathbf{G H z})\end{array}$ & $\begin{array}{l}\text { Return Loss } \\
(\mathbf{d B})\end{array}$ \\
\hline \multirow{3}{*}{$\begin{array}{c}\text { Without } \\
\text { Metamaterial }\end{array}$} & 1.054 & -17.611 \\
\cline { 2 - 3 } & 1.57 & -13.970 \\
\cline { 2 - 3 } & 1.949 & -17.007 \\
\cline { 2 - 3 } & 3.441 & -15.68 \\
\hline
\end{tabular}

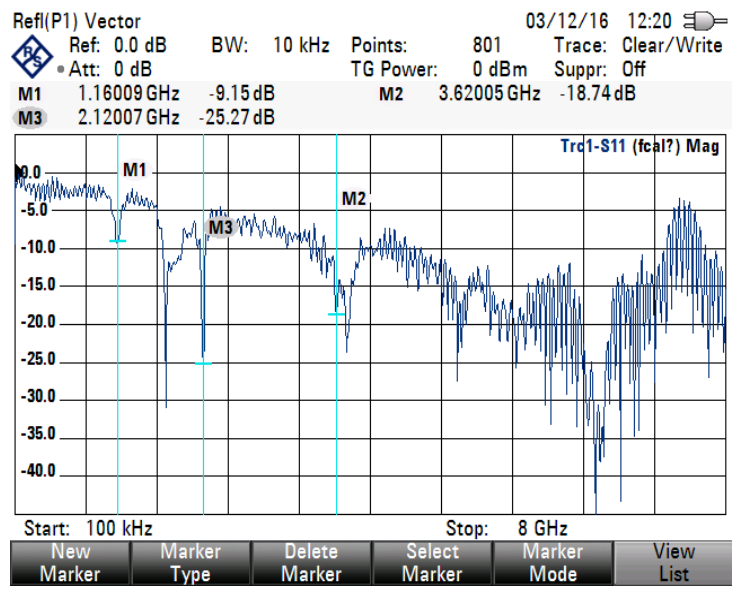

Figure 8 Measured result of sub patch 2

Table 2 Measured result for frequency and return loss of sub-patch 2 without loading

\begin{tabular}{|l|l|l|}
\hline & $\begin{array}{l}\text { Frequency } \\
(\mathbf{G H z})\end{array}$ & $\begin{array}{l}\text { Return Los } \\
(\mathbf{d B})\end{array}$ \\
\hline \multirow{2}{*}{$\begin{array}{l}\text { Without } \\
\text { Metamaterial }\end{array}$} & 1.16 & -9.15 \\
\cline { 2 - 3 } & 2.12 & -25.27 \\
\cline { 2 - 3 } & 3.62 & -18.74 \\
\hline
\end{tabular}

The simulated and measured return loss obtained is tabulated in table 1 and 2 .

To achieve high gain metamaterial is placed above microstrip patch antenna [3]-[5], as shown in Fig. 9. The results of the simulation and measured results are shown in the Fig. 10 and 11 resp.

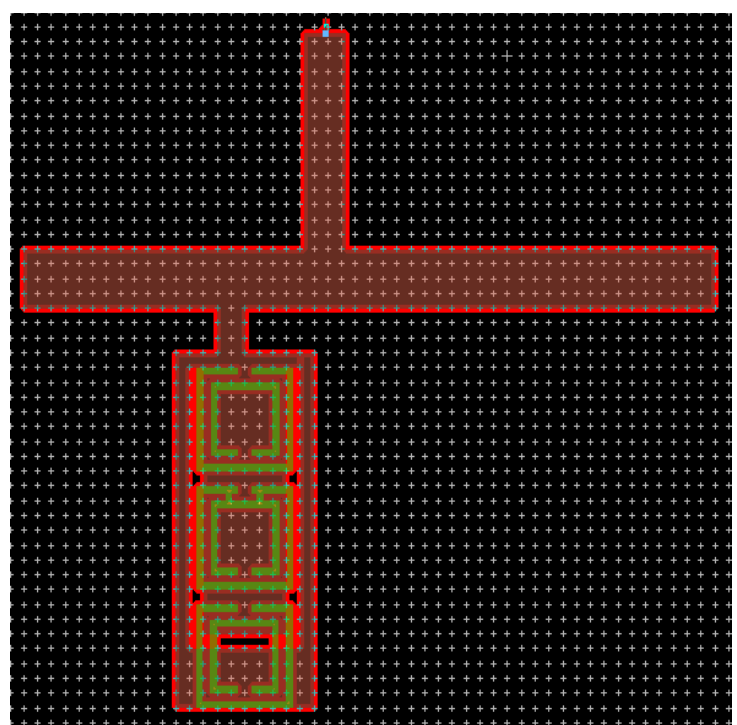

Figure 9 Sub-patch 2 with meta material loading 21 


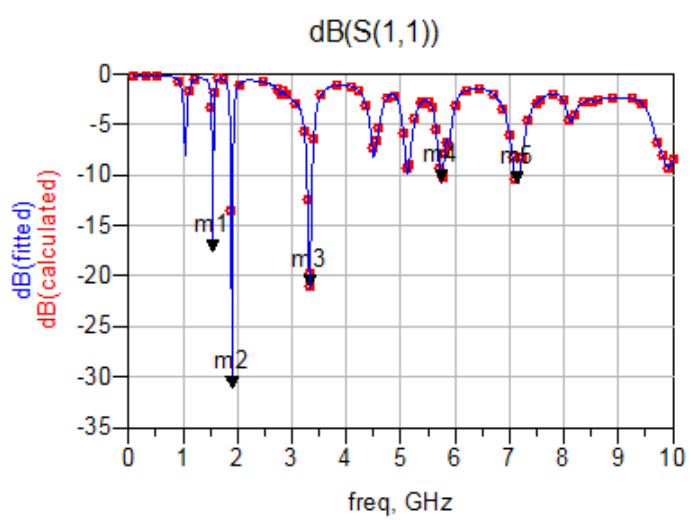

Figure 10 Simu lation results of sub-patch 2 with loading 21

Table 3 simulated result for frequency and return loss of sub-patch 2 with loading 21

\begin{tabular}{|l|l|l|}
\hline & $\begin{array}{l}\text { Frequency } \\
(\mathbf{G H z})\end{array}$ & $\begin{array}{l}\text { Return Loss } \\
(\mathbf{d B})\end{array}$ \\
\hline \multirow{4}{*}{ Load 21 } & 1.546 & -15.891 \\
\cline { 2 - 3 } & 1.897 & -31.051 \\
\cline { 2 - 3 } & 3.329 & -20.065 \\
\cline { 2 - 3 } & 5.751 & -10.661 \\
\cline { 2 - 3 } & 7.14 & -10.846 \\
\hline
\end{tabular}

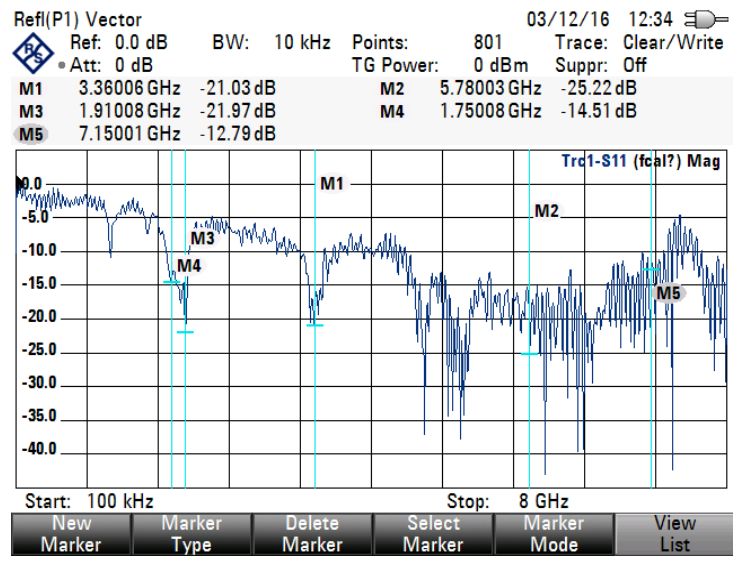

Figure 11 Measured result of sub patch 2 with loading 21

Table 4 measured result for frequency and return loss of sub-patch 2 with loading 21

\begin{tabular}{|l|l|l|}
\hline & $\begin{array}{l}\text { Frequency } \\
(\mathbf{G H z})\end{array}$ & $\begin{array}{l}\text { Return Loss } \\
(\mathbf{d B})\end{array}$ \\
\hline \multirow{4}{*}{ Load 21} & 1.75 & -14.51 \\
\cline { 2 - 3 } & 1.901 & -21.97 \\
\cline { 2 - 3 } & 3.36 & -21.03 \\
\cline { 2 - 3 } & 5.78 & -25.22 \\
\cline { 2 - 3 } & 7.15 & -12.79 \\
\hline
\end{tabular}

From Table 1, 2, 3 and 4, it is observed that return loss is more negative for MSA with metamaterial. Return loss indicate the gain, to achieve higher gain, return loss should be more negative. Further, the different loading helps in getting multiband operation. Thus the overall size of the patch is just equivalent to sub patch 2 [12].

\section{Conclusion}

The proposed microstrip antenna patch antenna with metamaterial gives a multiband operation, covering the frequency range of $\mathrm{L}, \mathrm{S}$ and $\mathrm{C}$ bands as compared to conventional microstrip patch antenna which cover only $\mathrm{L}$ and $\mathrm{S}$ bands. By analyzing the simulation result, it is found that the gain is also increased. Further the size of antenna is also reduced. Such a compact multiband microstrip antenna can be used for all the wireless applications thus saving space and cost.

\section{REFERENCES}

[1] Girish Kumar and K. P. Ray, broadband microstrip antenna (Artech House).

[2] V. I. Slyusar, Metamaterials on Antenna Solutions, in Proc. Intemational Conf. on Antenna Theory and Techniques, pp. 19-24, 6-9 Oct. 2009.

[3] H. A. Majid, M. K. A. Rahim, and T. Masri, Microstrip Antenna's Gain Enhancement Using Left Handed Metamaterial Structure, Progress In Electromagnetics Research M, Vol. 8, 235-247, 2009.

[4] Ahmad A. Sulaiman, Ahmad S. Nasaruddin, et.al., Bandwidth Enhancement in Patch Antenna by Metamaterial Substrate, European Journal of Scientific Research Vol.44 No.3 (2010), pp.493-501, 2010.

[5] R. K. Lou, T. Aribi, and C. Ghobadi, Improvement of Characteristics of Microstrip Antenna Using of Metamaterial Superstrate, in Proc. International Conf. on Communications Engineering, 22-24 Dec. 2010.

[6] George Casul, Cătălin Moraru, Andrei Kovacs, Design and Implementation of Microstrip Patch Antenna Array, IEEE, 2014

[7] V. Veselago, L. Braginsky, V. Shklover and C. Hafner, Negative Refractive Index Materials, $J$. Comput. Theor. Nanosci., Vol. 3, No. 2, 2006.

[8] Richard D. Averitt, introduction to metamaterials. Boston University, Boston

[9] Kyu-Chang LEE, Seung-In YANG, High Gain Patch Antenna for $2.4 \mathrm{GHz}$ using Metamaterial Superstrate, Supplement Journal of Measurement Science and Instrumentation, Vol.1, 2010.

[10] Adnan SONDAS, Mustafa Hikmet Bilgehan UCAR, Yunus Emre ERDEMLI, Tunable SRR-based substrate for a microstrip patch Antenna, Turk J Elec Eng \& Comp Sci, Vol.20, No.1, 2012.

[11] M. Borhani, P. Rezaei, and A. Valizade, Design of A Reconfigurable Miniat urized Microstrip Antenna for Switchable Multiband Systems, IEEE Antennas and Wireless Propagation, 2015.

[12] S. Dhamankar and U. P. Khot, "Design of Reconfigurable and Tunable Multiband Patch Antenna for Wireless Devices," in Proc. Intemational Conf. on Recent Advances \& Challenges in Engineering \& Management (RACEM-2014), Mumbai, 3-4, pp.1-3, April, 2014.

[13] Ning Zhu and Richard W. Ziolkowski, Active Met amaterial-Inspired Broad-Bandwidth, Efficient, Electrically Small Antennas, IEEE Antennas and Wireless Propagation Letters, VOL. 10, 2011.

[14] Ahmed Al-Shaheen, Enhancement Of Bandwidth Of Planar Microstrip Antenna With Metamaterials, ARPN Journal of Engineering and Applied Sciences, VOL. 6, No. 9, 2011. 